\title{
Role of Endoscopic Ultrasound-Guided Fine-Needle Aspiration of Portal Vein Thrombus in the Diagnosis and Staging of Hepatocellular Carcinoma
}

\author{
Dina Eskandere', Hazem Hakim ${ }^{1}$, Magdy Attwa', Wagdi Elkashef ${ }^{2}$ and Ahmed Youssef Altonbary ${ }^{1}$ \\ ${ }^{1}$ Department of Gastroenterology and Hepatology, Mansoura Specialized Medical Hospital, Mansoura University, Mansoura, \\ ${ }^{2}$ Department of Pathology, Mansoura University, Mansoura, Egypt
}

Background/Aims: Malignant portal vein thrombus (PVT) is found in up to $44 \%$ of patients with hepatocellular carcinoma (HCC). The nature of the thrombus influences treatment selection. The aim of this study was to assess the safety and efficacy of endoscopic ultrasound-guided fine-needle aspiration (EUS-FNA) in determining the nature of PVT in liver cirrhosis and/or HCC.

Methods: A prospective study was conducted in 34 patients with liver cirrhosis and/or HCC with PVT. Under EUS guidance, PVT was punctured using a 22 G FNA needle (Cook Medical, Bloomington, IN, USA) followed by monitoring of the puncture tract using color Doppler. Patients were followed for adverse events 2 hours after recovery.

Results: Throughout the 30-month study period, 34 patients, including 24 males with a mean age of $59 \pm 8$ years, were enrolled. There were 8 patients with known HCC and 26 with no liver masses detected by computed tomography (CT). EUS-FNA from PVT was positive for malignancy in 3 patients $(8.8 \%)$, of which only 1 patient was diagnosed with HCC by CT and 2 patients were newly diagnosed with HCC after EUS-FNA. No major complications were reported.

Conclusions: EUS-FNA is a safe and effective technique for determining the nature of PVT that does not fulfill the malignant criteria via imaging studies in patients with liver cirrhosis and/or HCC. Clin Endosc 2021;54:745-753

Key Words: Endoscopic ultrasound; Fine-needle aspiration; Hepatocellular carcinoma; Portal vein thrombus

\section{INTRODUCTION}

Portal vein thrombus (PVT) is increasingly being diagnosed. It has a wide spectrum of clinical presentation ranging from asymptomatic to potentially life-threatening conditions. It is commonly associated with abdominal tumors, liver cirrhosis, and hypercoagulable disorders. ${ }^{1}$ Malignant PVT is found in up to $44 \%$ of patients with hepatocellular carcinoma

Received: August 29, 2020 Revised: November 14, 2020

Accepted: December 9, 2020

Correspondence: Ahmed Youssef Altonbary

Department of Gastroenterology and Hepatology, Mansoura Specialized Medical Hospital, Mansoura University, Mansoura 35516, Egypt

Tel: +20-1005100091, Fax: +20-50-2222618, E-mail: a.tonbary@gmail.com

ORCID: https://orcid.org/0000-0001-8850-9829

(c) This is an Open Access article distributed under the terms of the Creative Commons Attribution Non-Commercial License (http://creativecommons.org/ licenses/by-nc/3.0) which permits unrestricted non-commercial use, distribution, and reproduction in any medium, provided the original work is properly cited.
(HCC). Nevertheless, bland PVT is found in up to $42 \%$ of patients with HCC and $26 \%$ of patients with liver cirrhosis. ${ }^{2}$ Not every PVT in a patient with HCC is a malignant thrombus; however, the nature of the thrombus eventually influences treatment selection. ${ }^{3}$ Moreover, PVT may even be the initial sign of an undetected HCC. ${ }^{4}$ Therefore, every effort should be made to differentiate between bland and malignant PVT.

Malignant PVT appears on imaging studies as a low-density plug with contrast enhancement in the arterial phase on both computed tomography (CT) and magnetic resonance imaging (MRI) and may have an arterial signal on Doppler ultrasound (US). However, bland PVT does not enhance with contrast or have any Doppler signal. ${ }^{3}$ Moreover, malignant PVT does not always exhibit neovascularity, which raises the need for fine-needle aspiration (FNA) to determine the nature of the PVT. $^{3}$ Percutaneous US-guided FNA of PVT may lead to false positive results because of unintended inclusion of normal hepatocytes or atypical cells from interposed liver masses. Fur- 
thermore, potential complications of percutaneous US-guided FNA of PVT include serious vascular and/or biliary injury. ${ }^{5}$

Unlike the percutaneous transhepatic approach, endoscopic ultrasound (EUS) offers a unique access and view of the main portal vein up to the porta hepatis. From the first part of the duodenum, the PVT can be punctured with an FNA needle under EUS guidance, avoiding interposing the bile duct, hepatic artery, and collateral circulation. In addition, the EUS-guided approach is supposed to have no false positive results as it eludes unintended inclusion of normal hepatocytes or atypical cells from interposed liver masses occurring with a percutaneous transhepatic approach. ${ }^{6}$ Most of the published literature describes percutaneous US-guided FNA of PVT, with only a few reports noting the use of EUS-FNA.

The aim of this study was to assess the feasibility, safety, and efficacy of EUS-FNA in determining the nature of PVT that did not fulfill the malignant criteria via imaging studies in patients with liver cirrhosis and/or HCC.

\section{PATIENTS AND METHODS}

\section{Patients}

This was a prospective study conducted in 34 patients with liver cirrhosis and/or HCC with PVT that did not fulfill the malignant criteria by triphasic abdominal CT, over a 30-month study period from May 2017 to December 2019 at the endoscopy unit of Mansoura Specialized Medical Hospital, Mansoura University (Egypt). The study protocol was approved by our ethical committee (Institutional Research Board of Mansoura University, no: 17.06.69), and written consent was obtained from all patients before the procedure.

\section{Inclusion criteria}

Patients with liver cirrhosis (with or without hepatic masses) and PVT that did not fulfill the criteria for malignancy by triphasic abdominal CT defined as: neovascularity of thrombus, arterial enhancement with rapid washout, or direct invasion by adjacent hepatic mass. ${ }^{2,3}$ Patients who underwent local or surgical treatment following a diagnosis of HCC and developed PVT during their follow-up.

\section{Exclusion criteria}

Patients with PVT fulfilling the malignant criteria by triphasic abdominal CT, patients with extrahepatic metastasis of HCC, patients with decompensated liver cirrhosis (ChildTurcotte-Pugh class C), patients with segmental branch PVT requiring traversing the hepatic parenchyma, patients with a contraindication to interventional endoscopy, such as those with coagulation disorders (International Normalized Ratio $\geq 1.5$, platelet count $\leq 50.000$ cells $/ \mathrm{mm}^{3}$ ), those unfit for sedation (ASA Class 3 or more), and those who refused to be involved in the study.

\section{Methods}

On the day of the procedure, eligible patients were appointed to the endoscopy room for EUS examination under intravenous propofol sedation. The patient's medical records were revised for the standard data, which included patient demographics, clinical history, laboratory investigations, major comorbidities that prevent curative management, imaging studies (abdominal US, triphasic abdominal CT, and chest X-ray) with special focus on the nature of PVT and the presence of liver masses. All EUS procedures were performed by two experienced endosonographers using a Pentax linear Echoendoscope EG3870UTK (PENTAX Medical, Tokyo, Japan) connected to a Hitachi Avius ultrasound system (Hitachi Medical Systems, Tokyo, Japan).

\section{Procedural technique}

Examination of the portal vein was performed from the first part of the duodenum with anticlockwise rotation following the portal vein up to the portal bifurcation at the liver hilum. Examination of the liver was performed from the fundus of the stomach with clockwise and anticlockwise rotation, inspecting most of the liver segments for any focal lesion. From the duodenal bulb, Color Doppler was used to identify the best position to puncture the portal vein, avoiding interposing blood vessels. Under EUS guidance, PVT was punctured using a 22 G FNA needle (Cook Medical, Bloomington). The needle was then moved to and fro 8-10 times while the stylet was slowly removed (slow pull technique). One or two passes were performed to avoid repeated punctures of the portal vein and decrease the risk of bleeding. Subsequently, the portal region was monitored using Color Doppler, and the puncture site was monitored endoscopically to identify any possible bleeding. The specimen was considered adequate using macroscopic on-site evaluation by obtaining a visible core $\geq 4 \mathrm{~mm}$. Tissue material was divided into two parts: the first one was placed in a formalin tube and the second was smeared onto slides and fixed with 95\% alcohol instantly labeled and name tagged. No pathologist was present onsite, and all samples were sent to the pathology department for evaluation. After recovery, patients were followed for adverse events for 2 hours and on the next day by phone.

\section{Cytopathological examination}

All slides were stained with hematoxylin and eosin, and all tissue samples fixed in formalin were placed in paraffin and stained with hematoxylin and eosin for evaluation of the pres- 
ence of a histologic core. Immunohistochemical markers were used when needed. All prepared slides and tissue samples were examined by an experienced cytopathologist.

\section{Statistical analysis}

The sample size was calculated using Power Analysis and Sample Size software program (PASS) version 11.0.4. Using the normal distribution curve, for an error of $2 \%$ and a level of confidence of $98 \%$, the sample size should be approximately 33 cases. A total of 34 patients were recruited in this study.

IBM's SPSS statistics (statistical package for the social science) for windows version 25 (SPSS Inc., Chicago, IL, USA) was used for statistical analysis of the collected data. Normally distributed continuous variables were expressed as mean \pm SD, whereas categorical variables and abnormally distributed continuous variables were expressed as median or number and percentage (as appropriate). As the actual sample size of the malignant PVT diagnosed by histopathology was found to be only 3 , the evaluation of $p$-value would be insignificant.

\section{RESULTS}

Throughout the 30-month study period, 45 patients were diagnosed with liver cirrhosis (with or without hepatic masses) and PVT that did not fulfill the malignant criteria by triphasic abdominal CT. Of these, 6 patients with coagulation disorders and 1 patient with small PVT in the segmental branch of the left portal vein (requiring traversing the hepatic parenchyma) were excluded. Four patients refused to undergo the procedure. Finally, 34 patients who fulfilled the inclusion criteria, including 24 men and 10 women with a mean age of $59 \pm 8$ years were enrolled. In 8 patients, liver masses were detected by triphasic abdominal CT with typical features of HCC (enhancement in the arterial phase and rapid washout in the portal and delayed phases); of these, 1 patient was shown to have malignant PVT by EUS-FNA. In 26 patients, no liver masses were detected by triphasic abdominal CT except for 1 patient with 2 small nodules $(15 \mathrm{~mm})$ in the right liver lobe with no enhancement in the arterial phase (diagnosed by CT as cirrhotic nodule), which proved to be malignant masses with malignant PVT after EUS-FNA. In the remaining 25 patients, a small focal lesion $(10 \mathrm{~mm}$ ) in the caudate lobe of the liver (not detected by triphasic abdominal CT) was detected by EUS and proved to be a malignant mass with malignant PVT in 1 patient. The other 24 patients were proved to be benign after EUS-FNA, similar to the CT results, as shown in the flowchart (Fig. 1).

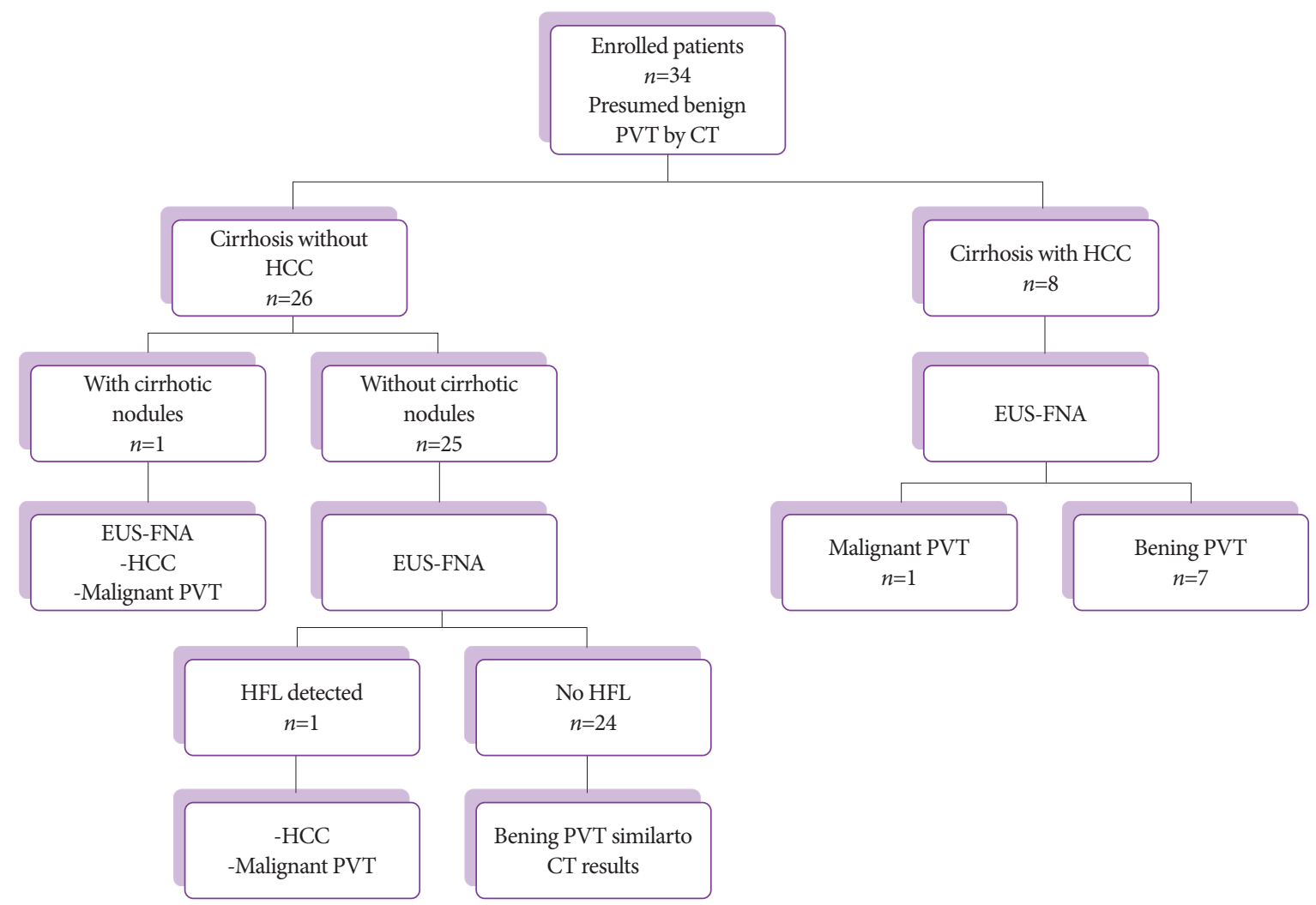

Fig. 1. Flowchart of enrolled patients. CT, computed tomography; EUS-FNA, endoscopic ultrasound-guided fine-needle aspiration; HCC, hepatocellular carcinoma; HFL, hepatic focal lesion; PVT, portal vein thrombus. 
EUS-FNA from PVT was positive for malignancy in 3 patients $(8.8 \%)$, of which only 1 patient was diagnosed with HCC by triphasic abdominal CT and 2 patients were newly diagnosed with HCC after EUS-FNA (from cirrhotic nodules in 1 patient and from a small lesion missed by CT in 1 patient). Patients were subdivided after the histopathological results of EUS-FNA into three groups: cirrhosis with benign PVT (24 patients), HCC with benign PVT (7 patients), and HCC with malignant PVT (3 patients). Details of demographic data, laboratory investigations, criteria of the PVT, presence of varices, description of HCC in enrolled patients and different scores used to assess the severity of liver disease and/or stage of HCC are shown in Tables 1-4.

EUS-FNA was technically feasible in all patients, despite the presence of portal cavernoma or collateral circulation in 16 patients (47\%). No major complications occurred in any patient. However, mild abdominal pain was observed in 4 patients (11.76\%) and mild self-limited bleeding at the puncture site was observed during endoscopic monitoring in 1 patient (2.94\%) (Table 5).

On review of the 34 aspirates, 3 were positive for malignancy and 31 were negative. Among the 3 positive cases, poorly differentiated adenocarcinoma with positive cytoplasmic reaction for HepPar-1 and Glypican-3 was seen in 1 patient (Fig. 2) and low-grade HCC with positive cytoplasmic reaction for HepPar-1 and Glypican-3 for both hepatic focal lesion and

Table 1. Demographic Data and Laboratory Investigations

\begin{tabular}{lccc}
\hline $\begin{array}{l}\text { Patients subdivi- } \\
\text { sions }\end{array}$ & $\begin{array}{c}\text { Cirrhosis } \\
\text { with benign } \\
\text { PVT } \\
\boldsymbol{n}=\mathbf{2 4} \\
\text { Mean (SD) }\end{array}$ & $\begin{array}{c}\text { HCC with } \\
\text { benign PVT } \\
\boldsymbol{n}=\mathbf{7} \\
\text { Mean (SD) }\end{array}$ & $\begin{array}{c}\text { HCC with } \\
\text { malignant } \\
\text { PVT } \\
\boldsymbol{n}=\mathbf{3} \\
\text { Mean (SD) }\end{array}$ \\
\hline Age & $58.3(8.4)$ & $58.8(7.5)$ & $64.67(2.1)$ \\
Gender & $\begin{array}{c}14 \text { males } \\
10 \text { females }\end{array}$ & 7 males & 3 males \\
Albumin $(\mathrm{g} / \mathrm{dL})$ & $3.2(0.4)$ & $3.3(0.2)$ & $3.2(0.3)$ \\
Bilirubin $(\mathrm{mg} / \mathrm{dL})$ & $2(2.4)$ & $0.9(0.2)$ & $1.7(0.6)$ \\
\hline INR & $1.2(0.1)$ & $1.2(0.1)$ & $1.2(0.1)$ \\
\hline SGOT $(\mathrm{U} / \mathrm{L})$ & $51.2(44.6)$ & $39.7(18.2)$ & $57.6(47.9)$ \\
\hline SGPT $(\mathrm{U} / \mathrm{L})$ & $31.9(25.7)$ & $25.5(11.1)$ & $37.6(18.1)$ \\
\hline WBC $\left(\times 10^{9} / \mathrm{L}\right)$ & $6.2(3.4)$ & $5.5(3.1)$ & $2.3(0.4)$ \\
\hline Hemoglobin $(\mathrm{g} / \mathrm{dL})$ & $10.2(2.1)$ & $11.5(2.7)$ & $9.1(0.7)$ \\
\hline Platelet $\left(\times 10^{9} / \mathrm{L}\right)$ & $143.2(110.2)$ & $124.1(61.8)$ & $63.3(2.8)$ \\
\hline
\end{tabular}

HCC, hepatocellular carcinoma; INR, international normalization ratio; PVT, portal vein thrombus; $\mathrm{SD}$, standard deviation; SGOT, serum glutamic-oxalacetic transaminase; SGPT, serum glutamic-pyruvic transaminase; WBC, white blood cells.
PVT was observed in 2 patients (Figs. 3 and 4). Of the 31 patients negative for malignancy, 1 patient showed suppurative smear with high cellularity formed mainly of neutrophils and clumps of pus cells.

A significant impact on the management plan was achieved after EUS-FNA in 4 patients (11.7\%). EUS-FNA upstaged 1 patient with HCC from Barcelona Clinic Liver Cancer (BCLC) stage B to stage $\mathrm{C}$ and from Hong Kong Liver Cancer (HKLC) stage IIIb to stage IVb, and the patient was treated with sorafenib instead of chemoembolization. In 2 patients, HCC was not diagnosed by triphasic abdominal CT and was diagnosed and staged after EUS-FNA as BCLC stage C and HKLC stage IVa and treated with sorafenib. In 1 patient, portal pyemia was diagnosed after EUS-FNA, which resolved completely on follow-up after treatment with antibiotics.

Table 2. Criteria of Portal Vein Thrombus and Presence of Varices

\begin{tabular}{|c|c|c|c|}
\hline Patients subdivisions & $\begin{array}{c}\text { Cirrhosis } \\
\text { with be- } \\
\text { nign PVT } \\
n=24\end{array}$ & $\begin{array}{c}\text { HCC with } \\
\text { benign } \\
\text { PVT } \\
n=7\end{array}$ & $\begin{array}{c}\text { HCC with } \\
\text { malignant } \\
\text { PVT } \\
n=3\end{array}$ \\
\hline Size mean (SD) & $20.1(4.8)$ & $17.1(5.2)$ & $23.5(3.9)$ \\
\hline \multicolumn{4}{|l|}{ Degree of Occlusion, $n(\%)$} \\
\hline Occlusive & $5(20.8)$ & $0(0)$ & $0(0)$ \\
\hline Non-occlusive & $19(79.2)$ & $7(100)$ & $3(100)$ \\
\hline \multicolumn{4}{|l|}{ Site, $n(\%)$} \\
\hline Only trunk & $14(58.3)$ & $3(42.9)$ & $0(0)$ \\
\hline Only branches & $2(8.3)$ & $1(14.3)$ & $0(0)$ \\
\hline Trunk \& one branch & $4(16.7)$ & $3(42.9)$ & $2(66.7)$ \\
\hline Trunk \& two branches & $4(16.7)$ & $0(0)$ & $1(33.3)$ \\
\hline \multicolumn{4}{|l|}{ Extent, $n(\%)$} \\
\hline Splenic vein & $2(8.3)$ & $0(0)$ & $0(0)$ \\
\hline Mesenteric vein & $5(20.8)$ & $0(0)$ & $0(0)$ \\
\hline Both of them & $4(16.7)$ & $1(14.3)$ & $0(0)$ \\
\hline Neither of them & $13(54.2)$ & $6(85.7)$ & $3(100)$ \\
\hline \multicolumn{4}{|l|}{ Duration, $n(\%)$} \\
\hline Recent & $8(33.3)$ & $4(57.1)$ & $0(0)$ \\
\hline Chronic & $16(66.7)$ & $3(42.9)$ & $3(100)$ \\
\hline \multicolumn{4}{|l|}{ Presence of varices, $n(\%)$} \\
\hline No varices & $6(25)$ & $1(14.3)$ & $0(0)$ \\
\hline Esophageal & $14(58.3)$ & $5(71.4)$ & $3(100)$ \\
\hline Fundic & $2(8.3)$ & $0(0)$ & $0(0)$ \\
\hline $\begin{array}{l}\text { Esophageal and fundic } \\
\text { varices }\end{array}$ & $2(8.3)$ & $1(14.3)$ & $0(0)$ \\
\hline
\end{tabular}

HCC, hepatocellular carcinoma; PVT, portal vein thrombus; SD, standard deviation. 
Table 3. Description of Hepatocellular Carcinoma in Enrolled Patients

\begin{tabular}{|c|c|c|c|c|c|c|}
\hline $\begin{array}{l}\text { The description of } \\
\text { the nature }\end{array}$ & $\begin{array}{c}\text { Previously } \\
\text { known as HCC }\end{array}$ & $\begin{array}{l}\text { EUS-FNA } \\
\text { of PVT }\end{array}$ & $\begin{array}{l}\text { Number } \\
\text { of lesions }\end{array}$ & Site of lesions & Size of lesions & $\begin{array}{c}\text { Previous } \\
\text { intervention } \\
\text { radiology }\end{array}$ \\
\hline Single nodule & Yes & Benign & 1 & Segment VIII & $1.2 \mathrm{~cm}$ & No \\
\hline Single nodule & Yes & Benign & 1 & Segment V & $1.5 \mathrm{~cm}$ & No \\
\hline Binodular & Yes & Benign & 2 & Segment V & 1.8 and $2 \mathrm{~cm}$, respectively & No \\
\hline Multinodular & Yes & Benign & 3 & $\begin{array}{l}\text { Segments VI ( } 2 \text { lesions), } \\
\text { VII ( } 1 \text { lesion })\end{array}$ & 3,4 and $2.6 \mathrm{~cm}$, respectively & TACE \\
\hline Multinodular & Yes & Benign & 5 & $\begin{array}{l}\text { Segments V (1 lesion), VI (3 } \\
\text { lesions), VII (1 lesion) }\end{array}$ & $\begin{array}{l}1.5,3.5,2.6,1.7 \text { and } 1.5 \mathrm{~cm} \text {, } \\
\text { respectively }\end{array}$ & TACE \\
\hline Multinodular & Yes & Benign & 4 & Segments II, V, VI, VII & $\begin{array}{l}4.8,1.5,5 \text { and } 2.5 \mathrm{~cm}, \\
\text { respectively }\end{array}$ & TACE \\
\hline Binodular & Yes & Benign & 2 & Segments V, VIII & 5.5 and $2.5 \mathrm{~cm}$ respectively & TACE \\
\hline Binodular & Yes & Malignant & 2 & Segments V, VIII & 5.3 and $2 \mathrm{~cm}$ respectively & No \\
\hline $\begin{array}{l}\text { Single nodule (Missed } \\
\text { lesion by CT) }\end{array}$ & No & Malignant & 1 & $\begin{array}{l}\text { Segment I } \\
\text { Caudate lobe }\end{array}$ & $1 \mathrm{~cm}$ & No \\
\hline $\begin{array}{l}\text { Binodular (Cirrhotic } \\
\text { nodules by CT) }\end{array}$ & No & Malignant & 2 & Segment V & $1.5 \mathrm{~cm}$ & No \\
\hline
\end{tabular}

CT, computed tomography; EUS-FNA, endoscopic ultrasound-guided fine-needle aspiration; HCC, hepatocellular carcinoma; PVT, portal vein thrombus; TACE, trans-arterial chemoembolization

Table 4. Different Scores Used to Assess Severity of Liver Disease and/or Stage of Hepatocellular Carcinoma

\begin{tabular}{|c|c|c|c|}
\hline Patients subdivisions & $\begin{array}{c}\text { Cirrhosis with benign PVT } \\
\begin{array}{c}n=24 \\
n(\%)\end{array}\end{array}$ & $\begin{array}{c}\text { HCC with benign PVT } \\
n=7 \\
n(\%)\end{array}$ & $\begin{array}{c}\text { HCC with malignant PVT } \\
\qquad \begin{array}{c}n=3 \\
n(\%)\end{array}\end{array}$ \\
\hline \multicolumn{4}{|l|}{ СТP } \\
\hline $\mathrm{A}$ & $14(58.3)$ & $4(57.1)$ & $2(66.7)$ \\
\hline $\mathrm{B}$ & $10(41.7)$ & $3(42.9)$ & $1(33.3)$ \\
\hline \multicolumn{4}{|l|}{ BCLC staging } \\
\hline Very early stage (0) & - & $2(28.6)$ & $0(0)$ \\
\hline Early stage (A) & - & $1(14.3)$ & $0(0)$ \\
\hline Intermediate (B) & - & $4(57.1)$ & $0(0)$ \\
\hline Advanced (C) & - & $0(0)$ & $3(100)$ \\
\hline \multicolumn{4}{|l|}{ HKLC staging } \\
\hline Stage I & - & $2(28.6)$ & $0(0)$ \\
\hline Stage IIa & - & $1(14.3)$ & $0(0)$ \\
\hline Stage IIIb & - & $4(57.1)$ & $0(0)$ \\
\hline Stage IVa & - & $0(0)$ & $2(66.6)$ \\
\hline Stage IVb & - & $0(0)$ & $1(33.3)$ \\
\hline
\end{tabular}

BCLC, Barcelona Clinic Liver Cancer; CTP, Child-Turcotte-Pugh; HCC, hepatocellular carcinoma; HKLC, Hong Kong Liver Cancer; PVT, portal vein thrombus. 
Table 5. Procedure Related Complications

\begin{tabular}{lccc} 
& Cirrhosis with benign PVT & HCC with benign PVT & \multicolumn{2}{c}{ HCC with malignant PVT } \\
Patients subdivisions & $\boldsymbol{n}=\mathbf{2 4}$ & $\boldsymbol{n}=\mathbf{7}$ & $\boldsymbol{n}(\%)$ \\
& $\boldsymbol{n}(\mathbf{\%})$ & $\boldsymbol{n}(\%)$ & $1(33.3)$ \\
\hline Pain & $3(12.5)$ & $0(0)$ & $0(0)$ \\
Bleeding at puncture site & $1(4.2)$ & $0(0)$ & $2(66.7)$ \\
\hline No complications & $20(83.3)$ & $7(100)$ & $(\%)$ \\
\hline
\end{tabular}

HCC, hepatocellular carcinoma; PVT, portal vein thrombus.
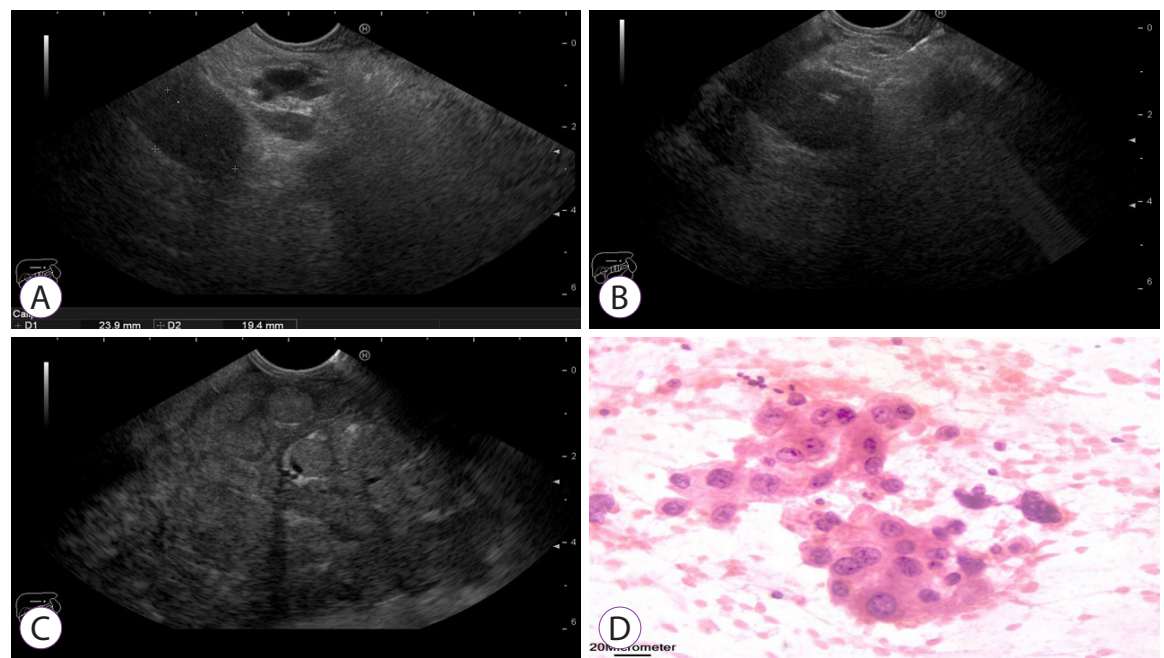

Fig. 2. (A) Endoscopic ultrasound examination showing hyperechoic thrombus totally obstructing the main portal vein. (B) Endoscopic ultrasound-guided fine-needle aspiration using $22 \mathrm{G}$ needle for Portal vein thrombus. (C) Multiple small hepatic focal lesions. (D) Cytopathological examination showing sheets of malignant cells with moderate atypia, prominent nucleoli, and occasional mitotic figures, consistent with poorly differentiated adenocarcinoma.
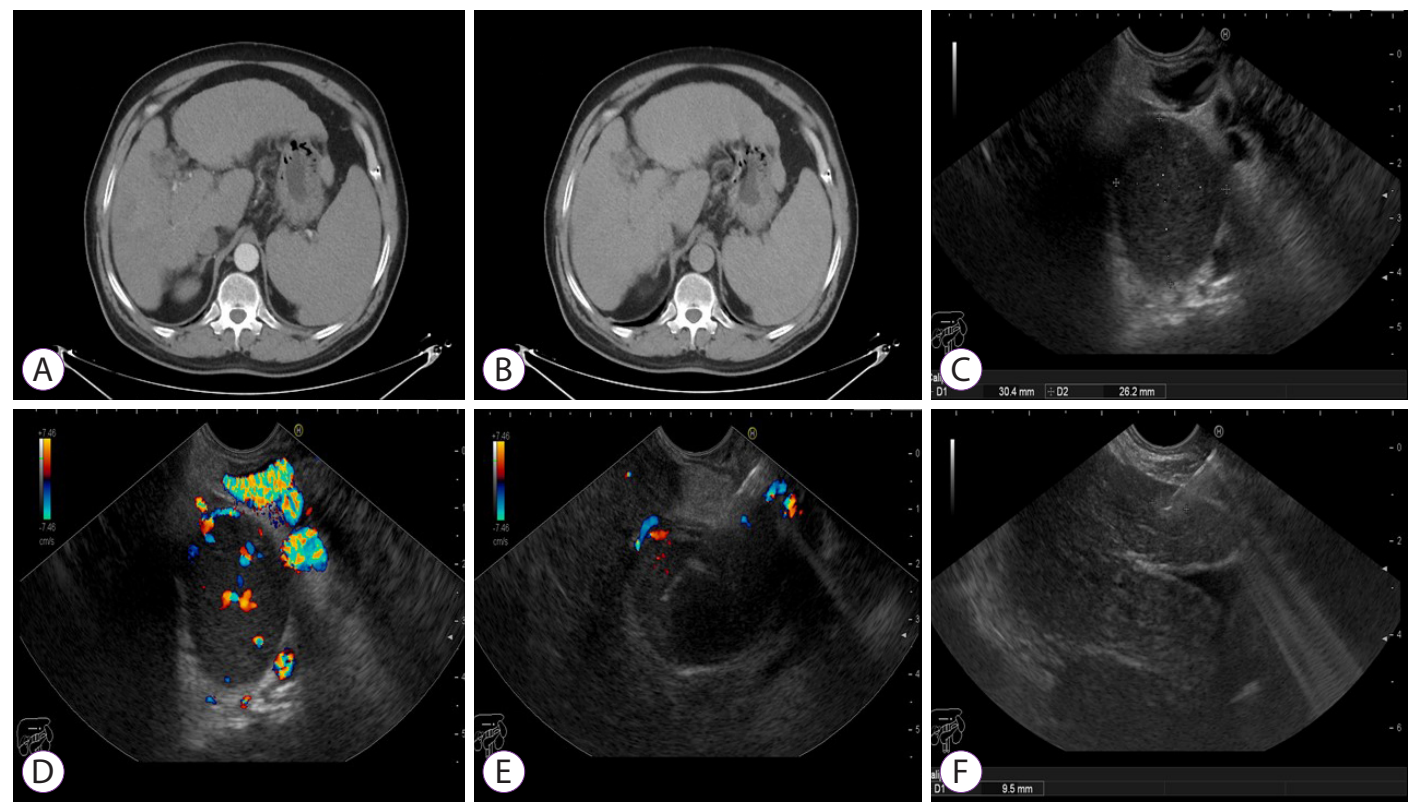

Fig. 3. (A. B) Triphasic computed tomography showing non-enhancing portal vein thrombus with no definite liver masses. (C, D) Endoscopic ultrasound examination showing hyperechoic thrombus partially obstructing the main portal branch with no doppler flow inside and multiple periportal veins (cavernoma). (E, F) Endoscopic ultrasound-guided fine-needle aspiration using $22 \mathrm{G}$ needle for both Portal vein thrombus and hepatic focal lesion. 

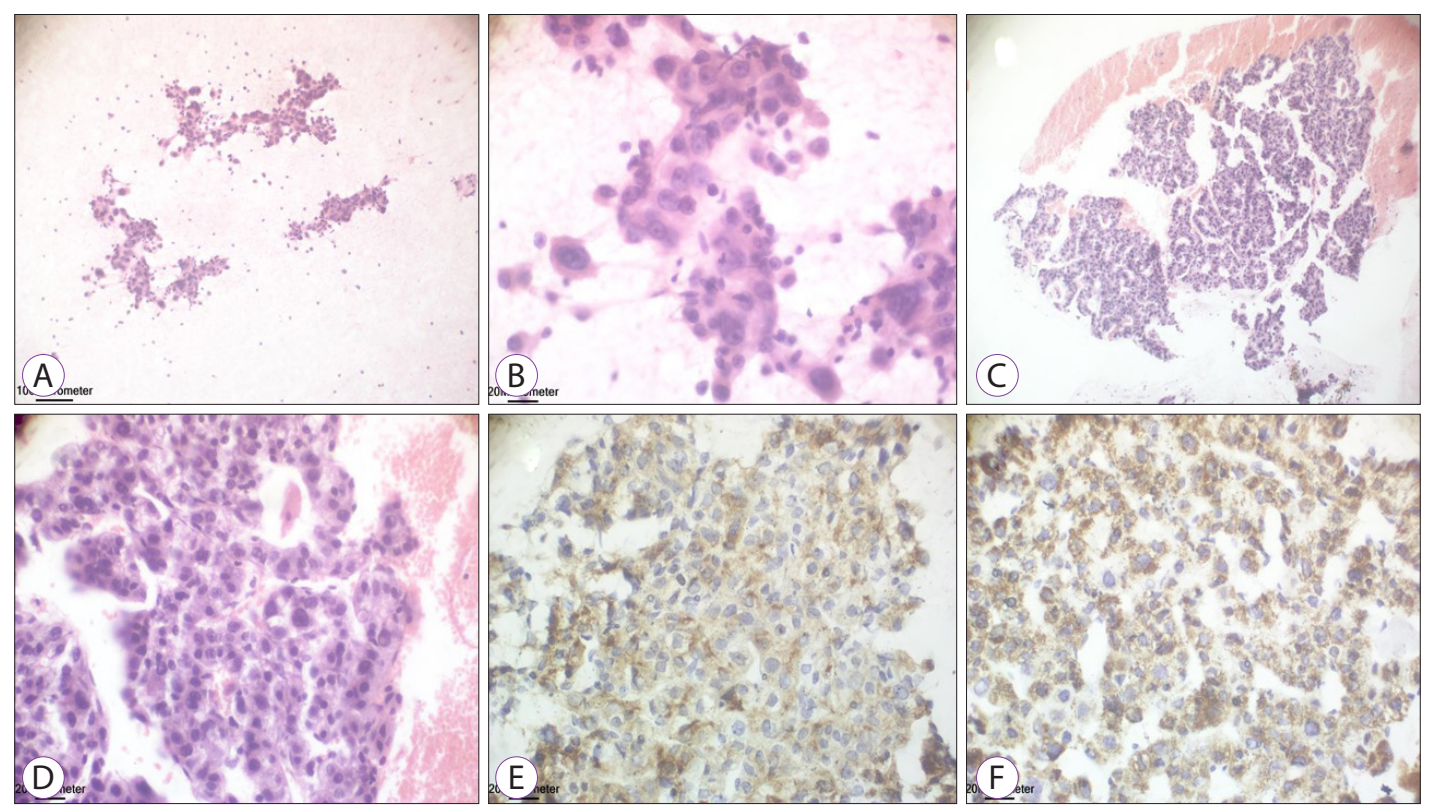

Fig. 4. Cytopathological examination showing sheets of malignant cells with moderate nuclear atypia with granular eosinophilic cytoplasm (A, B) PVT. (C, D) Hepatic focal lesion. (E, F) Special stain shows positive cytoplasmic reaction for HepPar-1 and Glypican-3, consistent with low-grade hepatocellular carcinoma.

\section{DISCUSSION}

HCC is one of the most common malignant tumors worldwide and accounts for approximately $90 \%$ of primary liver tumors. ${ }^{7}$ HCC invasion into the portal vein or extrahepatic vascular metastasis affects patient survival and changes the approach of management by precluding many treatment modalities, such as liver transplantation, surgical resection, ablation, or chemoembolization, which are usually associated with a high recurrence rate. Therefore, the recognition of PVT and accurate differentiation of bland from malignant thrombus are crucial for patient management. ${ }^{8}$

Non-invasive imaging studies, such as contrast-enhanced CT or MRI are currently used in the diagnosis of HCC, although they have some limitations. HCC is typically hypervascular on contrast-enhanced CT and shows notable enhancement on arterial phase, with rapid washout on the portal and delayed phases. ${ }^{7}$ Whenever a PVT shows contrast enhancement in the arterial phase, it is considered a malignant thrombus until proven otherwise. However, these non-invasive studies have their fallacies as malignant PVT does not always exhibit neovascularity, and recanalizing bland PVT may exhibit the flow inside. ${ }^{9}$ In a study by Teama et al., ${ }^{10}$ who evaluated the efficacy of contrast-enhanced CT and Doppler US in differentiating bland from malignant PVT, 16 patients with malignant PVT proved by FNA were included. Neovas- cularization and enhancement in the arterial phase on contrast-enhanced CT were detected in 14 patients with a sensitivity of $87.5 \%$, and arterial signal by Doppler US was detected in 12 patients with a sensitivity of $70 \%$. Malignant PVT was not diagnosed by either contrast-enhanced CT or Doppler US in 2 patients, which is consistent with the results of our study, where 3 patients with PVT that did not fulfill the malignant criteria by triphasic abdominal CT were diagnosed as malignant thrombus only after EUS-FNA.

Percutaneous transhepatic sampling of a PVT for the diagnosis of HCC was first described by Joly et al. in $1993 .{ }^{11}$ HCC was diagnosed in this case by percutaneous transhepatic US-guided biopsy from the left PVT after failure of both US and CT to detect any hepatic mass. Subsequently, several case series reported the use of this technique in determining the nature of PVT and in staging of HCC. ${ }^{6}$ In a study by Rammohan et al., ${ }^{8} 20$ patients with PVT and confirmed or suspicious HCC underwent US-guided FNA of PVT. Liver masses were detected by imaging studies in 17 patients before US-guided FNA of PVT. In the remaining 3 patients, HCC was diagnosed after US-guided FNA of PVT in 2 patients, although no liver masses were detected by imaging studies before FNA. On evaluation of the 20 aspirates, malignancy was confirmed in 16 aspirates as follows: poorly differentiated carcinoma in 3, moderately differentiated HCC in 10, and well-differentiated HCC in 3 , and 4 aspirates were negative. In our study, malignancy 
was confirmed in 3 aspirates as follows: poorly differentiated adenocarcinoma with positive cytoplasmic reaction for HepPar-1 and Glypican-3 in 1 aspirate and low-grade HCC with positive cytoplasmic reaction for HepPar-1 and Glypican-3 for both hepatic focal lesions and PVT in 2 aspirates, while 31 aspirates were negative. However, this study did not exclude the malignant criteria of PVT by imaging studies before FNA as in our study, which could explain the high percentage of positive malignant aspirates in their study compared to ours, which showed only 3 malignant PVTs out of 34 aspirates.

Despite its well-established efficacy, percutaneous transhepatic US-guided FNA of PVT is not widely utilized, which could be explained by many rationales. First, this technique is associated with complications, such as biliary injury, vascular injury, arteriovenous or vascular-biliary fistula formation, and pseudoaneurysm formation. ${ }^{12}$ Second, technical difficulties may be experienced either in obese patients or in targeting centrally located PVT while avoiding an interposed hepatic mass that requires maximal needle excursion. Finally, this technique requires traversing the hepatic parenchyma with inclusion of hepatocytes in the sample, leading to false positive results. ${ }^{6}$ Theoretically, EUS can overcome these difficulties with the proximity of the echoendoscope to the portal vein from the duodenal bulb, avoiding traversing the hepatic parenchyma and any interposed vascular or biliary structure, making the procedure precise and quick with minimal complications. ${ }^{13}$

EUS-FNA of PVT for the diagnosis of HCC was first described by Lai et al. in 2004. ${ }^{6}$ HCC was diagnosed in this case by EUS-FNA of PVT after misinterpretation of a right lobe liver mass detected by $\mathrm{CT}$ as a combination of hepatic changes related to PVT and biliary dilation. This was followed by 4 case reports describing the use of EUS-FNA of PVT in the diagnosis and staging of suspected or confirmed HCC., ${ }^{3,13-15}$ In a retrospective study by Rustagi et al. ${ }^{16}$ evaluating the efficacy and safety of EUS-FNA using 22 and $25 \mathrm{G}$ needles from a remote malignant thrombus (vascular thrombus away from the primary tumor), 17 patients were included. Malignancy was confirmed after EUS-FNA in 12 patients (70.6\%) as follows: 5 pancreatic adenocarcinomas, 2 cholangiocarcinomas, 2 neuroendocrine tumors, 1 lung cancer, 1 lymphoma, and 1 HCC. In this study, EUS-FNA was upstaged in 5 patients with pancreatic adenocarcinoma without any reported complications.

In another retrospective study by Gimeno Garcia et al. ${ }^{17}$ evaluating the feasibility and safety of EUS-FNA of PVT associated with HCC, 8 patients were included out of 23 candidate patients. EUS-FNA using 22 and $25 \mathrm{G}$ needles was technically feasible in 7 patients with no reported complications and confirmed malignancy in 6 of them (87.5\%) who were upstaged as follows: 2 patients with BCLC stage A, 1 with BCLC stage B, and 3 in whom HCC was not previously diagnosed; all 6 were upstaged to BCLC stage C. The high percentage of positive malignant aspirates (6 out of 7 patients) in this study could be explained by the dropped-out patients. As a result of the retrospective design of this study, 15 candidate patients were not referred for EUS-FNA, and 1 patient was excluded due to technical difficulties in performing EUS-FNA in the presence of interposing collateral vessels.

In our study, EUS-FNA upstaged 1 patient with HCC from BCLC stage B to stage $C$ and from HKLC stage IIIb to stage $\mathrm{IVb}$, and the patient was treated with sorafenib instead of chemoembolization. In 2 patients, HCC was not diagnosed by triphasic abdominal CT and was diagnosed and staged after EUS-FNA as BCLC stage C and HKLC stage IVa and treated with sorafenib. To the best of our knowledge, this is the first prospective study evaluating the feasibility and efficacy of EUS-FNA in determining the nature of PVT that did not fulfill the malignant criteria via imaging studies in patients with liver cirrhosis and/or HCC. The main limitation of our study was the relatively small number of enrolled patients. Larger studies are required to confirm our results.

In conclusion, EUS-FNA is a safe and effective technique for determining the nature of PVT that does not fulfill the malignant criteria via imaging studies in patients with liver cirrhosis and/or HCC. The integration of EUS-FNA in the evaluation process of HCC may influence treatment selection. We believe that EUS-FNA should be utilized more frequently in the staging of HCC and in diagnosing the etiology of PVT.

Conflicts of Interest

The authors have no potential conflicts of interest.

Funding

None.

ORCID
Dina Eskandere:

Hazem Hakim:

Magdy Attwa:

Wagdi Elkashef:

Ahmed Youssef Altonbary: https://orcid.org/0000-0001-5324-7670 https://orcid.org/0000-0001-8273-7871 https://orcid.org/0000-0003-2600-8003 https://orcid.org/0000-0001-8850-9829 https://orcid.org/0000-0001-9073-4830

\section{REFERENCES}

1. Handa P, Crowther M, Douketis JD. Portal vein thrombosis: a clinician-oriented and practical review. Clin Appl Thromb Hemost 2014;20:498-506

2. Catalano OA, Choy G, Zhu A, Hahn PF, Sahani DV. Differentiation of malignant thrombus from bland thrombus of the portal vein in patients with hepatocellular carcinoma: application of diffusion-weighted MR 
imaging. Radiology 2010;254:154-162.

3. Michael H, Lenza C, Gupta M, Katz DS. Endoscopic ultrasound -guided fine-needle aspiration of a portal vein thrombus to aid in the diagnosis and staging of hepatocellular carcinoma. Gastroenterol Hepatol (N Y) 2011;7:124-129.

4. Tarantino L, Ambrosino P, Di Minno MN. Contrast-enhanced ultrasound in differentiating malignant from benign portal vein thrombosis in hepatocellular carcinoma. World J Gastroenterol 2015;21:9457-9460.

5. ASGE Technology Committee; Trikudanathan G, Pannala R, et al. EUS-guided portal vein interventions. Gastrointest Endosc 2017;85:883888.

6. Lai R, Stephens V, Bardales R. Diagnosis and staging of hepatocellular carcinoma by EUS-FNA of a portal vein thrombus. Gastrointest Endosc 2004;59:574-577.

7. Coakley FV, Schwartz LH. Imaging of hepatocellular carcinoma: a practical approach. Semin Oncol 2001;28:460-473.

8. Rammohan A, Jeswanth S, Sukumar R, et al. Percutaneous ultrasound-guided fine-needle aspiration of portal vein thrombi as a diagnostic and staging technique for hepatocellular carcinoma. Abdom Imaging 2013;38:1057-1060.

9. Tarantino L, Francica G, Sordelli I, et al. Diagnosis of benign and malignant portal vein thrombosis in cirrhotic patients with hepatocellular carcinoma: color Doppler US, contrast-enhanced US, and fine-needle biopsy. Abdom Imaging 2006;31:537-544.

10. Teama AH, Elbarbary AA, Elhawary KE, Abusekina WA. Color Doppler US and tri-phasic CT in differentiating benign from malignant portal vein thrombosis (PVT). The Egyptian Journal of Radiology and Nuclear
Medicine 2015;46:847-857.

11. Joly JP, Delamarre J, Razafimahaleo A, Sevestre H, Tossou H, Capron JP. Occult hepatocellular carcinoma in cirrhosis: value of ultrasound-guided biopsy of portal vein system thrombus. Abdom Imaging 1993;18:344346.

12. Dodd GD 3rd, Carr BI. Percutaneous biopsy of portal vein thrombus: a new staging technique for hepatocellular carcinoma. AJR Am J Roentgenol 1993;161:229-233.

13. Kayar Y, Turkdogan KA, Baysal B, Unver N, Danalioglu A, Senturk H. EUS-guided FNA of a portal vein thrombus in hepatocellular carcinoma. Pan Afr Med J 2015;21:86.

14. Storch I, Gomez C, Contreras F, Schiff E, Ribeiro A. Hepatocellular carcinoma (HCC) with portal vein invasion, masquerading as pancreatic mass, diagnosed by endoscopic ultrasound-guided fine-needle aspiration (EUS-FNA). Dig Dis Sci 2007;52:789-791.

15. Moreno M, Gimeno-García AZ, Corriente MM, et al. EUS-FNA of a portal vein thrombosis in a patient with a hidden hepatocellular carcinoma: confirmation technique after contrast-enhanced ultrasound. Endoscopy 2014;46 Suppl 1:E590-E591.

16. Rustagi T, Gleeson FC, Chari ST, et al. Remote malignant intravascular thrombi: EUS-guided FNA diagnosis and impact on cancer staging. Gastrointest Endosc 2017;86:150-155.

17. Gimeno Garcia AZ, Aparicio JR, Barturen A, Moreno M, Nicolas-Perez D, Quintero E. Endoscopic ultrasound-guided fine-needle aspiration of portal vein thrombosis in patients with chronic liver disease and suspicion of hepatocellular carcinoma. Eur J Gastroenterol Hepatol 2018;30:418-423. 